\title{
Comparison of CCS and Toast Classifications with Single Center Cases in Determining Etiological Stroke Subtypes
}

ISSN: 2637-7748

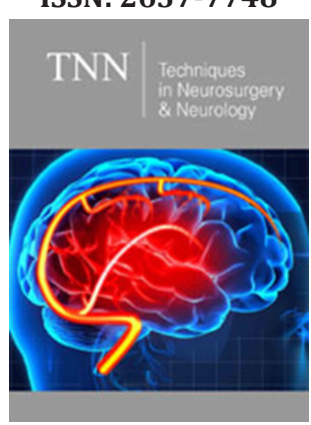

*Corresponding author: Erkan Acar, Department of Neurology, Turkey

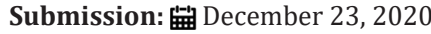

Published: March 23, 2021

Volume 4 - Issue 1

How to cite this article: Erkan Acar, Zeynep Özdemir, Betül Yalçıner, Göksel Bakaç, Aysun Soysal. Comparison of CCS and Toast Classifications with Single Center Cases in Determining Etiological Stroke Subtypes. Tech Neurosurg Neurol. 4(1). TNN. 000580. 2021.

DOI: 10.31031/TNN.2021.04.000580

Copyright@ Erkan Acar, This article is distributed under the terms of the Creative Commons Attribution 4.0 International License, which permits unrestricted use and redistribution provided that the original author and source are credited.
Erkan Acar ${ }^{1}$, Zeynep Özdemir ${ }^{2}$, Betüll Yalçıner ${ }^{3}$, Göksel Bakaç ${ }^{4}$ and Aysun Soysal $^{2}$

${ }^{1}$ Department of Neurology, Turkey

${ }^{2}$ Osman Education and Research Hospital for Psychiatric and Neurological Diseases, Turkey

${ }^{3}$ Bayındır Hospital, Turkey

${ }^{4}$ Şişli Florence Nightingale Hospital, Turkey

\section{Abstract}

Background: Identifying the pathophysiological mechanism of stroke is important for proper treatment. Causative Classification of Stroke (CCS) and Trial of ORG 10172 in Acute Stroke Treatment (TOAST) are the mostly used classifications for maintaining underlying pathophysiology.

Method: Documents of 2096 patients who were hospitalized with Ischemic Stroke (IS) and Transient Ischemic Attack (TIA) between 2002-2005 were reviewed retrospectively. Classifications were applied to 459 patients and others were excluded because of inadequate data. Underlying mechanisms were determined by using CCS and TOAST. We compared the classifications to each other according to the etiological findings of patients.

Objective: We aimed to find out the rates of etiologies in our study group and if one classification is superior to others to reduce the undetermined case ratio.

Result: There were 394 IS and 65 TIA patients. 9.7\% of IS, 32.3\% of TIA according to CCS whereas $20.1 \%$ of IS, $86.2 \%$ of TIA according to TOAST were assigned as undetermined ( $\mathrm{p}<0.001$ ). $16 \%$ Cardioembolism (CE), 50.8\% large artery atherosclerosis (LAA), 10.2\% small artery occlusion (SAO), 13.5\% More Than One Cause (MTOC) according to CCS, whereas $14.7 \%$ CE, 42.9\% LAA, 5.6\% SAO, 16\% MTOC according to TOAST were reported in IS \%) ( $\mathrm{p}<0.001,0.684,0.031,0.024,0.374$, respectively). $9.2 \%$ CE, 47.7\% LAA, $4.6 \%$ SAO and 6.2\% MTOC were found according to CCS, whereas 1.5\% CE, 9.2\% LAA ( $\mathrm{p}<0.001$ ), $1.5 \%$ SAO and $1.5 \%$ MTOC according to TOAST were reported in TIA.

Conclusion: Most frequent etiology was LAA in both CCS and TOAST, whereas the least frequent were other causes. We thought that CCS reduces the proportion of undetermined etiology than TOAST in patients. We need further studies for appropriate results.

\section{Introduction}

The ischemic stroke (IS) and transient ischemic attack (TIA) etiologies are various, so it's difficult to classify into subgroups [1]. Causative Classification of Stroke (CCS) [2] and Trial of Org 10172 in Acute Stroke Treatment (TOAST) [3,4] are the mostly used classifications. TOAST assigns ischemic strokes to five subtypes based upon clinical features and the results of ancillary studies including brain imaging, neurovascular evaluations, cardiac tests, and laboratory evaluations for a prothrombotic state. CCS is a computerized algorithm that consists of a questionnaire-style classification scheme. Classifying into subgroups is vital to maintain underlying pathophysiology and decide the accurate treatment, thereby patients could be prevented from a secondary stroke. Similar categories (Large Artery Atherosclerosis (LAA), Cardioembolism (CE), Small Artery Occlusion More Than One Cause (SAO), (MTOC), Undetermined Causes (UC), and Other Etiology) are used in CCS [5] and TOAST [2] by using different criteria and data. However, after TOAST was developed in 1993, there have been many progresses in the field of magnetic resonance imaging which have resulted in determining the etiology of several undetermined underlying pathologies of stroke. More recently CCS has been developed to evaluate these grey zone patients [2]. In this review, we describe the etiology of stroke in a Turkish population and compare these two classification 
systems to find if there is a difference in subgroup ratios and one is superior to others to reduce the undetermined population.

\section{Methods}

We analyzed documents of 2096 patients who were hospitalized With Ischemic Stroke (IS) and Transient Ischemic Attack (TIA) between 2002-2005 retrospectively. Classifications were applied to 459 patients and others were excluded because of inadequate data. Deficits lasting less than 24 hours with negative imaging findings were considered as TIA, those with positive imaging findings with any duration of symptoms were considered as stroke. Clinical, laboratory and imaging data of patients were collected and evaluated. Demographic characteristics, stroke risk factors, presenting symptoms, and diagnostic test findings were used to classify patients. These data were used to determine etiologic subtypes by using CCS and TOAST classification systems to compare each other. The TOAST system divides the etiology of the stroke into five categories based on clinical impression and diagnostic test findings: cardioembolism, supra-aortic large artery atherosclerosis, small vessel occlusion, other causes, and undetermined etiology.

The CCS system is a web-based semiautomated algorithm that integrates clinical, imaging, and laboratory information. The five categories valid for the TOAST system is the same for the CCS system. The algorithm classifies each of the subtypes as 'evident', 'probable', and 'possible'. In this study, we didn't consider subtypes of the classification system. Statistical analyses were performed by using MedCalc Statistical Software version 12.7.7. Descriptive statistics were used to describe continuous variables (mean, median, standard deviation, minimum, maximum). Chi-square or Fisher Exact tests were used to examine the relationship between categorical variables. $\mathrm{P}<0.05$ was considered to be statistically significant. The primary analysis comprised of determining the frequencies of etiologic subgroups of TIA and IS patients within both TOAST and CCS classifications. This study was conducted in accordance with ethical principles and the study protocol was approved by the Ethics Committee of Bakırköy Prof. Dr. Mazhar Osman Training and Research Hospital. It was not considered necessary to get consent from the patients because the study was a retrospective data analysis.

\section{Result}

Data of 459 patients (199(43.4\%) female and 260(56.6\%) male) who admitted to our neurology clinic between 2002 and 2005 years were collected. Ages were between 31 and 95 (mean; 67.16 \pm 12.3 ). There were 394 (85.8\%) IS and 65 TIA patients (14.2\%) (Tables 1 \& 2). In IS group 106 (27\%) had smoking history, 107 (28.7\%) had DM, 59 (15.0\%) had CAD, 280 (71.4\%) had HT, 139 (35.4\%) had HL and 86 (22\%) had AF. In TIA group the numbers were 17 (26.1\%), 15 (23.1\%), 13 (20\%), 43 (66.1\%), 23 (35.4\%) and 10 (15.3\%) (Figure 1). The analyze of all patients showed that undetermined ratio was $12.9 \%(n=59)$ according to CCS whereas $29.4 \%(n=135)$ according to TOAST (p<0.001). 69 (15\%) CE, 231 (50.3\%) LAA, 43 (9.4\%) SAO, 57 (12.4\%) MTOC were found for CCS. The corresponding proportions for TOAST were 59 (12.9\%), 175 (38.1\%), 23 (5\%), 64 (13.9\%) ( $\mathrm{p}=0.415,0.003,0.014$ and 0.566 , respectively) (Figure 2). When we investigated IS patients, $38(9.7 \%)$ undetermined cases, 63 (16\%) CE, 200 (50.8\%) LAA, 40 (10.2\%) SAO, 53 (13.5\%) MTOC were found according to CCS. The corresponding proportions for TOAST were 79 (20.1\%), 58 (14.7\%), 169 (42.9\%), 22 (5.6\%), 63 (16\%) ( $\mathrm{p}<0.001,0.684,0.031,0.024,0.374$, respectively) (Figure $3)$. When we investigated TIA patients, 21 (32.3\%) undetermined cases, 6 (9.2\%) CE, 31 (47.7\%) LAA, 3 (4.6\%) SAO, 4 (6.2\%) MTOC were found according to CCS. The corresponding proportions for TOAST were $56(86.2 \%)(\mathrm{p}<0.01), 1(1.5 \%), 6$ (9.2\%) $(\mathrm{p}<0.01), 1$ (1.5\%), $1(1.5 \%)$ respectively (Figure 4$)$.

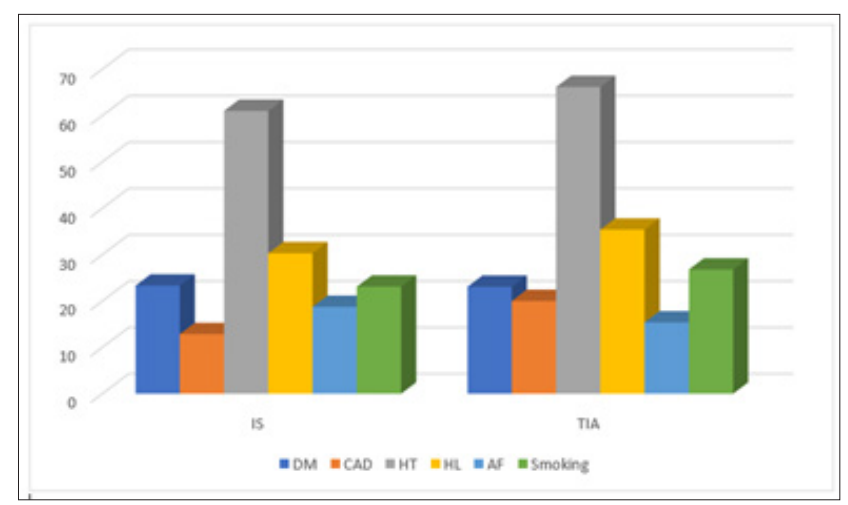

Figure 1: Risk factors of IS and TIA patients.

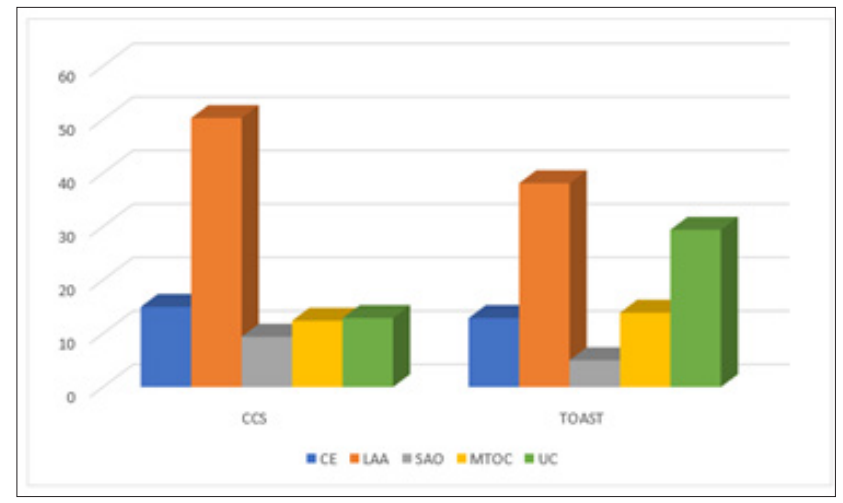

Figure 2: Classification of all patients.

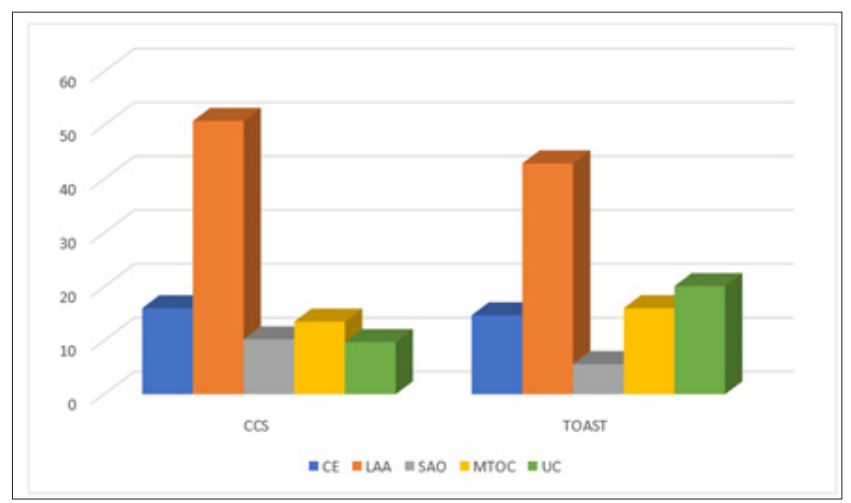

Figure 3: Classification of IS patients. 


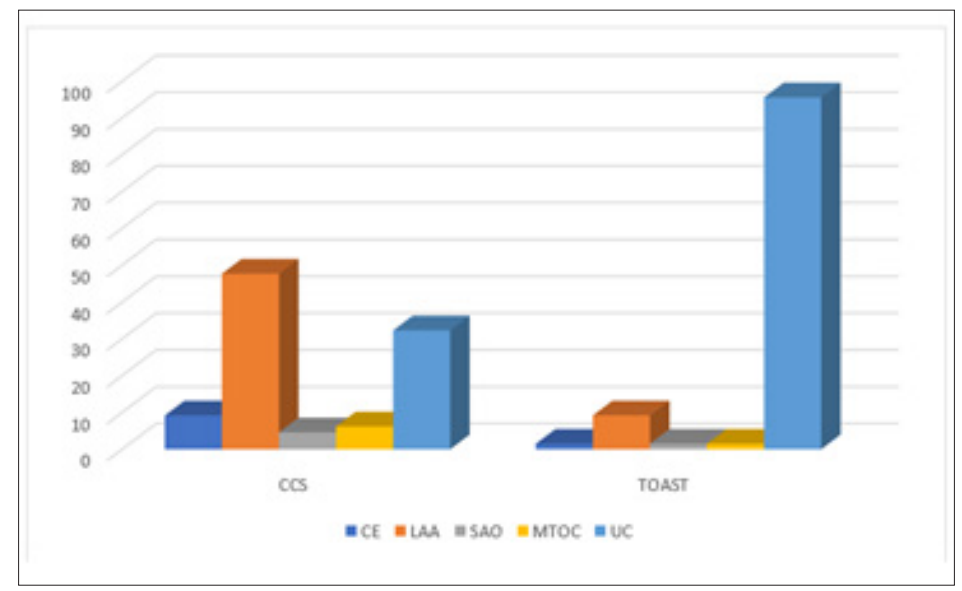

Figure 4: Classification of TIA patients

Table 1: Demographic features of patients.

\begin{tabular}{|c|c|}
\hline Characteristic & No. (\%) \\
\hline Age (y) mean \pm SD & $67.16 \pm 12.3$ \\
\hline (min-max) & $(31-95)$ \\
\hline Sex n (\%) & $199(43.36 \%)$ \\
Female & $260(56.64 \%)$ \\
Male & $122(26.57 \%)$ \\
\hline Risk Factors & $323(70.37 \%)$ \\
DM & $162(35.29)$ \\
HT & $72(15.68)$ \\
HL & $96(20.91)$ \\
CAD & $123(26.79 \%)$ \\
\hline
\end{tabular}

Table 2: Demographic features according to IS or TIA groups.

\begin{tabular}{|c|c|c|c|}
\hline \multirow{3}{*}{ Sex } & & IS n(\%) & TIA n(\%) Total n(\%) \\
\hline & Female & $174(37.91 \%)$ & $25(38.46 \%) 199(43.36 \%)$ \\
\hline & Male & $220(47.93 \%)$ & $40(61.54 \%) 260(56.64 \%)$ \\
\hline \multirow{6}{*}{ Risk Factors } & $\mathrm{DM}$ & $107(23.31 \%)$ & $15(23.07 \%) 122(26.57 \%)$ \\
\hline & HT & $280(61 \%)$ & $43(66.15 \%) 323(70.37 \%)$ \\
\hline & HL & $139(30.28 \%)$ & $23(35.38 \%) 162(35.29)$ \\
\hline & CAD & $59(12.85 \%)$ & $13(20 \%) 72(15.68)$ \\
\hline & $\mathrm{AF}$ & $86(18.73 \%)$ & $10(15.38 \%) 96(20.91)$ \\
\hline & Smoking & $106(23.09 \%)$ & $17(26.15 \%) 123(26.79 \%)$ \\
\hline \multirow{5}{*}{ CCS } & Cardioaortic Embolism & $63(16 \%)$ & $6(9.2 \%) 69(15.03 \%)$ \\
\hline & Supra-Aortic Large Artery Atherosclerosis & $200(43.57 \%)$ & $31(47.7 \%) 231(50.32 \%)$ \\
\hline & Small Artery Occlusion & $40(8.71 \%)$ & $3(4.6 \%) 43(9.37 \%)$ \\
\hline & Other causes & $53(11.54 \%)$ & $4(6.2 \%) 57(12.41 \%)$ \\
\hline & Undetermined Unknown - İncomplete Evaluation & $38(8.27 \%)$ & $21(32.3 \%) 59(12.85 \%)$ \\
\hline
\end{tabular}




\begin{tabular}{|c|c|c|c|}
\hline \multirow{5}{*}{ TOAST } & Cardioaortic Embolism & $58(12.69 \%)$ & $1(1.5 \%) 59(12.85 \%)$ \\
\hline & Supra-Aortic Large Artery Atherosclerosis & $169(36.81 \%)$ & $6(9.2 \%) 175(38.12 \%)$ \\
\hline & Small Artery Occlusion & $22(4.79 \%)$ & $1(1.5 \%) 23(5.01 \%)$ \\
\hline & Other causes & $63(13.72 \%)$ & $1(1.5 \%) 64(13.94 \%)$ \\
\hline & Undetermined Unknown incomplete Evaluation & $79(17.21 \%)$ & $56(86.2 \%) 135(29.41 \%)$ \\
\hline
\end{tabular}

\section{Discussion}

TOAST and CCS are widely used classification systems to determine ischemic stroke subgroups based on neurological symptoms, brain imaging, and adjunct tests. The CCS system is a web-based semiautomated algorithm that was developed to come through the major limitations of TOAST [6]. This study assessed the comparison of CCS and TOAST classification systems in a stroke population in Turkey. In the present study, the incidence of subgroups is large artery atherosclerosis, cardioembolism, small artery occlusion, multiple causes, and other causes from highest to lowest respectively for both classifications. In consideration of our findings, CCS has been found more successful to reduce undetermined/cryptogenic subgroup rate than TOAST which is crucial to managing treatment. When we investigate the subgroups in the ischemic stroke population, cardioembolism rate is higher in CCS than TOAST. In another population-based study, this ratio is $30.2 \%$ [7]. Cardiogenic embolism is the cause in about $15-20 \%$ of all ischemic stroke patients [8]. These differences may be due to incomplete evaluation of patients and undetermined cases, but our study showed similar cardioembolic rates to the general population. Large artery atherosclerosis and small artery occlusion are also determined in higher incidences in CCS like cardioembolism. In the TIA group, mostly found etiology is LAA for CCS and unknown causes for TOAST. Unknown etiology is in second, CE is in third and MTOC is in the fourth rank for CCS. LAA is the second most found etiology for TOAST, after that CE, SAO, MTOC were in the same rank. CE, LAA, SAO, and MTOC rates are higher in CCS than TOAST. There is a significant difference between the two systems in undetermined cases. This difference may be due to incomplete evaluation or insufficient patient population, beside this CCS may be accepted more successfully to reduce the undetermined ratios in TIA groups. In another study about the etiological classification of TIA, the undetermined rates were lower in total, but similar in CCS and TOAST systems. The mostly found subgroups were CE,
LAA, and SAO, respectively. In the present study, we think that CCS reduces the proportion of undetermined etiology than TOAST in patients. However, differences exist between the two systems in our study population, which merit further study.

\section{Authorship Contribution}

Surgical and Medical Practices: BY, GB, Concept: EA, ZÖ, BY, GB, AS, Design: EA, ZÖ, AS, Data Collection or Processing: EA, BY, GB, AS, Analysis or Interpretation: EA Literature Search: EA, ZÖ, AS, Writing: EA, ZÖ, AS.

\section{References}

1. Muir KW (2002) Heterogeneity of stroke pathophysiology and neuroprotective clinical trial design. Stroke 33(6): 15451550.

2. Adams HP, Bendixen BH, Kappelle LJ (1993) Classification of subtype of acute ischemic stroke: definitions for use in a multicenter clinical trial. TOAST. Trial of Org 10172 in acute stroke treatment. Stroke 24(1): 3541.

3. Ay H, Furie KL, Singhal A (2005) An evidence-based causative classification system for acute ischemic stroke. Ann Neurol 58(5): 688697.

4. Arsava EM, Ballabio E, Benner T (2010) The causative classification of stroke system: an international reliability and optimization study. Neurology 75(14): 1277-1284.

5. Ay H, Benner T, Arsava EM (2007) A computerized algorithm for etiologic classification of ischemic stroke: the causative classification of stroke system. Stroke 38(11): 2979-2984.

6. Chen PH, Shan Gao, Yong Jun W (2012) Classifying ischemic stroke, from TOAST to CISS. CNS Neurosciences and Therapeutics 18(6): 452-456.

7. Marnane M, Duggan CA, Sheehan OC (2010) Stroke subtype classification to mechanism-specific and undetermined categories by TOAST, AS-C-O, and causative classification system: direct comparison in the north dublin population stroke study. Stroke 41(8): 1579-1586.

8. Kolominsky RPL, Weber M, Gefeller O (2001) Epidemiology of ischemic stroke subtypes according to TOAST criteria: incidence, recurrence, and long-term survival in ischemic stroke subtypes: a population-based study. Stroke 32(12): 2735-2740. 